\title{
La importancia de la normalización para el ejercicio profesional del archivista
}

\author{
Manuela Moro Cabero* \\ Dunia Llanes Padrón**
}

Artículo recibido:

17 de noviembre de 2015

Artículo aceptado:

27 de septiembre de 2016

\section{Resumen}

La archivística posmoderna está marcada por la influencia directa de estándares que orientan y regulan el quehacer de los archivos así como la gestión documental. En los últimos años, los procesos técnicos, la administración de servicios y unidades de información, los sistemas de gestión de documentos y la preservación de recursos se apoyan en un conjunto de normas que facilitan su ejecución de forma sistemática, uniforme y eficaz. Este trabajo tiene como propósito identificar, describir y categorizar las normas destinadas a la representación de contenidos y su recuperación, a la continuidad y preservación de recursos digitales, a la verificación de calidad, a la excelencia empresarial y a los sistemas de gestión de documentos.

* Universidad de Salamanca, España._moroca@usal.es

** Universidad de La Habana, Cuba. duniallp@yahoo.es

INVESTIGACIÓN BIBLIOTECOLÓGICA, vol.32, núm.74, enero/marzo, 2018, México, ISSN: 2448-8321 pp. 193-223 
El estudio que se presenta es de naturaleza descriptiva centrado en la identificación de dicha normativa con atención en su aplicación en los currículos de los archivistas. La sociedad moderna demanda profesionales que sean capaces de actuar en contextos de trabajo diferentes y posean las habilidades para implementar operaciones que engloban los procesos y la gestión de información. Como consecuencia, se debe modelar el perfil del profesional y fundamentar su trabajo en patrones normalizados y homogéneos que recojan todo tipo de prácticas.

Palabras clave: Normalización; Preservación digital; Representación y recuperación de contenidos; Calidad y excelencia empresarial; Gestión de documentos

The importance of standardization in the professional practice of the archive specialist Manuela Moro-Cabero, Dunia LLanes-Padrón

\section{Abstract}

The postmodern Archivology exhibits the influence of standards that guide and regulate file and records management. Nowadays, the technical processes of information and documentation, services management, information centers and records management systems are founded on standards that allow systematic, uniform and effective operations. The aim of this work is to identify, describe and categorize the standards addressing content representation and retrieval, and the continuity and preservation of digital resources, the quality review of systems, business excellence and records management systems. Largely descriptive, this study focuses on identification of these standards and their implementation in Archivology curricula. The modern society requires professionals who are able to work in diverse workplace environments, possessed of the skills needed to implement all manner of operations and processes entailed in information management. As a result, the professional profile of the Archive specialist should match standardized, homogeneous models that include all kinds of practices.

Keywords: Standardization; Digital preservation; Representation and retrieve content; Quality and excellence management; Records management 


\section{INTRODUCCIÓN}

- esde las postrimerías del pasado siglo y hasta nuestros días, el ejercicio de archivar se ha visto auxiliado por un conjunto de normas que facilitan el tratamiento técnico de la información y de los documentos así como la administración de los servicios, procesos, programas, unidades de información y sistemas de gestión para los documentos. Los entornos digitales han aportado mayor complejidad al tratamiento y preservación de los recursos, razón por la que la práctica de archivar se ha tornado más dificultosa exigiendo para su correcto desempeño, a nuestro entender, un ejercicio analítico y sistemático, tanto de representaciones de contenidos (descripción, metadatos, indización, clasificación) como de su organización y administración (mediante el conocimiento de los contextos, la gestión, implementación de sistemas y programas para los documentos, el análisis y gestión de riesgos vinculados a los recursos de archivo gestionados o conservados, el control de estrategias de preservación, la gestión de la interoperabilidad y de la seguridad de la información, la administración de servicios y de unidades de información), sin obviar la evaluación y certificación de repositorios, servicios, procesos y sistemas, entre otros.

En la comunidad archivística se han empleado numerosas normas destinadas a la representación de los contenidos desde la década de 1980, con un alcance internacional y nacional. Esta labor ha facilitado el desarrollo e implementación de una normativa heterogénea de finalidades divergentes, aunque con un alcance internacional, como son los marcos International Organization for Standardization (ISO) o normas del Consejo Internacional de Archivos (CIA) o, incluso, normas específicas de aplicación (meta) nacional (marco de fomento de la e-administración e interoperabilidad). La cuantía de estándares que el archivista debe aplicar ante dichos contextos digitales de trabajo se ha incrementado considerablemente en un plazo muy reducido. Su alcance, contenido e interrelación deberían conocerse, así como dominar su aplicabilidad para facilitar su trabajo.

En este sentido, el estudio que se presenta es de naturaleza descriptiva y se centra en la identificación de dicha normativa en atención al contexto de su aplicación con la intención de presentarla de modo sistematizado y de resaltar su importancia. Dada su notabilidad, se pretende invitar al lector a reflexionar sobre la necesidad de su presencia en los currículos de los profesionales de la comunidad archivística, considerando su referencia en los planes de estudio del área profesional, dado que, tal y como se refleja en el Libro blanco para el diseño de titulaciones universitarias en el marco de la economía digital (España. Ministerio de Industria, Energía y Turismo, 2015: 17): “El 
nuevo ecosistema digital se presenta como un importante desafío en el que se redefinen las bases de la innovación, de la productividad, así como los modelos educativos y de empleo", exigiendo a los mismos completar, modificar y cambiar sus competencias de aprendizaje. El ensayo se estructura aglutinando la utilidad de la normativa analizada del siguiente modo: normas destinadas a la representación de contenidos y su recuperación, normas vinculadas a la continuidad y preservación de recursos digitales y normas marco MSS empleadas tanto para construir y verificar calidad y excelencia empresarial como sistemas de gestión normalizados para los documentos. Se pretende con la presente identificación y categorización destacar su utilidad así como la necesidad de su implementación en los planes de estudios para formar al futuro profesional con esta orientación; a su vez, se busca establecer una propuesta formativa e investigadora extensible al conjunto de normas identificadas. Este ensayo forma parte de una investigación comparada de mayor alcance donde se analiza la presencia de dichas normas en los títulos vinculados al área profesional y se establece una propuesta de competencias, objetivos y contenidos curriculares formativos para su aprendizaje y presencia en las titulaciones. En este sentido, algunos de sus resultados en lo referente a los contenidos y competencias a adquirir en estudios universitarios de Brasil y España han sido presentados en el IV Seminario Hispano-Brasileño (Llanes-Padrón y Moro-Cabero, 2015) y se está trabajando para concretar información comparada en Iberoamérica y Europa, siendo financiado por la Fundación Carolina, desde 2016.

\section{NORMALIZAR PARA REPRESENTAR Y RECUPERAR LOS CONTENIDOS}

La presencia de la normalización en la representación y recuperación de la información, en el quehacer de los archivos, es un acontecimiento bastante reciente. La regulación de estos procesos es el resultado de los cambios ocurridos en esta ciencia que transita, en la actualidad, por los caminos del paradigma posmoderno. Durante muchos años la descripción archivística fue considerada un proceso técnico y se asociaba a la elaboración de instrumentos de búsqueda (guías, catálogos, inventarios, índices).

Los instrumentos de búsqueda o referencia resultantes del proceso de representación no se realizaban de forma homogénea; cada sistema de archivo diseñaba los instrumentos con estructuras de datos propias, adecuadas a sus realidades archivísticas, por tanto, era imposible pensar en un intercambio de información entre instituciones diferentes. 
Los profesionales del área no aceptaban la idea de normalizar los procesos archivísticos, mucho menos la descripción. Los archiveros defendían ciertas premisas relacionadas con las características del documento de archivo, el perfil único de los usuarios o la forma tradicional de trabajo en los archivos que dificultaban el inicio de cualquier proceso normativo. Algunos investigadores manifestaban que la representación de la información no podía ser normalizada a nivel internacional pues bajo ningún concepto se podían realizar para los archivos normas semejantes a las existentes en las bibliotecas para catalogar.

Estas discusiones fueron superadas y los archiveros, finalmente, comprendieron la necesidad de cambiar los métodos usados en el proceso de descripción pues, sin lugar a dudas, este constituía el camino más eficaz para obtener mejores resultados en la representación de las entidades archivísticas y su intercambio en los ambientes digitales. Como bien plantea Rodríguez García (2012: 164):

[... Los fundamentos de la práctica descriptiva de 1960 al 2000 se transformaron considerablemente, no sólo por la rápida proliferación de nuevos tipos de publicación, nuevas formas de contenido y nuevos formatos, sino también porque la automatización, el uso de la tecnología y el actual movimiento de las redes en línea modificaron el trabajo de las instituciones de información y la interacción con los usuarios.

El evento que marca el cambio irreversible en las concepciones de la representación y la recuperación de la información en los archivos fue el proceso de normalización desarrollado por el Consejo Internacional de Archivos (CIA). En la década de 1990, el CIA desarrolló varias actividades que dieron lugar a la publicación de su conjunto normativo. En la actualidad, los profesionales disponen de cuatro normas internacionales para la descripción: la Norma Internacional para la Descripción Archivística (ISAD-G), la Norma Internacional sobre los Registros de Autoridades de Archivos relativos a Instituciones, Personas y Familias (ISAAR-CPF), la Norma Internacional para la Descripción de Funciones (ISDF) y la Norma Internacional para Describir Instituciones que Custodian Fondos de Archivo (ISDIAH).

El CIA se basó, para la creación de estos estándares, en las relaciones que se establecen entre productor, funciones, documentos e instituciones de custodia. A partir de esta interpretación de la realidad archivística, creó estructuras de datos internacionales adaptadas a cada una de las entidades. El proceso de normalización internacional no tuvo por objetivo regular los contenidos de la descripción a nivel internacional. Como consecuencia, las 
comunidades y asociaciones nacionales de archivos tenían la responsabilidad de elaborar normas propias de descripción para normalizar los contenidos de acuerdo a las realidades archivísticas nacionales.

A partir de esta premisa, y motivados por las ventajas y las perspectivas de la normalización de la descripción, varios países desarrollaron normas nacionales y regionales de contenidos, presentación y codificación para describir documentos, crear registros de autoridades y normalizar la selección de puntos de acceso. Gran Bretaña, Estados Unidos, Canadá, Portugal, España, Irlanda, Brasil y Uruguay son los países, que hasta la fecha, han elaborado y publicado estándares descriptivos de este tipo. El Manual of Archival Description (MAD3), Describing Archives: a Content Standards (DACS), las Rules for Archival Description (RAD2), las Orientações para a Descrição Arquivística (ODA), la Norma Brasileira de Descrição Arquivística (NOBRADE), el Manual de Descripción Multinivel (MDM), la Norma Española de Descripción Archivística - Borrador (NEDAI), la Norma de Descripción Archivística de Cataluña (NODAC), la Norma Gallega de Descripción Archivística (NOGADA), la Norma Aragonesa para la Descripción de Autoridades de Archivos (ARANOR), la Norma Irlandesa de Descripción (IGAD) y la Norma Uruguaya de Descripción Archivística (NUDA) son algunas de las normas publicadas en estos países.

La archivística experimentó, en pocos años, un cambio sustancial distanciándose de la inactividad en la regulación del proceso de descripción para potenciar la acción normativa. Como resultado, actualmente nos encontramos con un abanico de siglas que nombran los estándares utilizados por los archiveros para regular la descripción. Los proyectos normativos publicados buscan alcanzar modelos que se ajusten a las tradiciones archivísticas y que sistematicen los procedimientos e instrumentos de descripción existentes en cada país. Todo esto hace que, aunque partan de un texto común (normas internacionales), existan diferencias entre las distintas normas publicadas.

A través de la mayoría de estas normas se pueden representar los documentos de archivos, no sucediendo lo mismo con los productores, las funciones, las instituciones de custodia, los documentos especiales o la normalización de puntos de acceso. La Tabla 1 es un referente y ejemplifica las entidades archivísticas que describen las normas publicadas.

\begin{tabular}{|l|c|c|c|c|c|}
\hline \multicolumn{7}{|c|}{ Entidades que describen las normas } \\
\hline & Documento & Productor & $\begin{array}{c}\text { Documentos } \\
\text { especiales }\end{array}$ & Funciones & $\begin{array}{c}\text { Institución } \\
\text { custodia }\end{array}$ \\
\hline ISAD(G) & $X$ & - & - & - & - \\
\hline ISAAR(CPF) & - & $X$ & - & - & - \\
\hline
\end{tabular}




\begin{tabular}{|l|c|c|c|c|c|}
\hline ISDF & - & - & - & $x$ & - \\
\hline ISDIAH & - & - & - & - & $X$ \\
\hline MAD3 & $X$ & - & $X$ & - & - \\
\hline DACS & $X$ & $X$ & - & - & - \\
\hline RAD2 & $X$ & - & $X$ & - & - \\
\hline ODA & $X$ & $X$ & - & - & - \\
\hline NOBRADE & $X$ & - & - & - & - \\
\hline NEDAI & $X$ & - & - & - & - \\
\hline MDM & $X$ & $X$ & $X$ & - & - \\
\hline NODAC & $X$ & - & - & - & - \\
\hline NOGADA & $X$ & - & - & - & - \\
\hline ARANOR & - & $X$ & - & - & - \\
\hline IGAD & $X$ & - & - & - & - \\
\hline NUDA & $X$ & - & - & - & - \\
\hline
\end{tabular}

Tabla 1. Entidades presentes en las normas de descripción

Fuente: elaborada por las autoras

Tal y como se puede observar en la Tabla 1, en la mayoría de las normas se han desarrollado las estructuras de datos y contenidos para describir documentos. ISAAR (CPF),DACS, ODA, MDM y ARANOR son las únicas normativas que permiten, también, la descripción de los productores y proporcionan las pautas para la creación de los registros de autoridades. MAD3, RAD2 y el MDM desarrollan estructuras para la descripción de materiales en soportes especiales (fotografías, planos, documentos cartográficos, sonoros, películas, objetos, documentos filatélicos, electrónicos, entre otros). Sólo las propuestas internacionales incluyen estructuras para representar las funciones y las instituciones de custodia.

Estos estándares, atendiendo a su tipología, se pueden clasificar según su función en normas de entrada de datos y normas de salida de datos. El archivero español Abelardo Santamaría Gallo establece que las normas de entrada de datos se clasifican en normas de estructura de datos y de contendido mientras que las normas de salida de datos son categorizadas en normas de presentación y codificación (Santamaría Gallo, 2006: 12-13).

Reflejo de estas jerarquías es la Tabla 2 donde se muestra la tipología normativa a la que pertenece la nueva generación de estándares publicados. 


\begin{tabular}{|c|c|c|c|c|}
\hline \multicolumn{5}{|c|}{ Tipología de norma según función } \\
\hline & Estructura & Contenido & Presentación & Codificación \\
\hline $\operatorname{ISAD}(G)$ & $X$ & - & - & - \\
\hline ISAAR(CPF) & $x$ & - & - & - \\
\hline ISDF & $x$ & - & - & - \\
\hline ISDIAH & $x$ & - & - & - \\
\hline MAD3 & $x$ & $x$ & $x$ & $X$ \\
\hline DACS & $x$ & $x$ & - & $x$ \\
\hline RAD2 & $x$ & $x$ & $x$ & - \\
\hline ODA & $x$ & $x$ & $x$ & - \\
\hline NOBRADE & $x$ & $x$ & - & - \\
\hline NEDAI & $x$ & $x$ & $x$ & - \\
\hline MDM & $x$ & $x$ & $x$ & - \\
\hline NODAC & $x$ & $X$ & - & - \\
\hline NOGADA & $x$ & $x$ & - & - \\
\hline ARANOR & $x$ & $x$ & - & - \\
\hline IGAD & $x$ & $x$ & - & - \\
\hline NUDA & $x$ & $x$ & $x$ & - \\
\hline
\end{tabular}

Tabla 2. Clasificación de las normas según su función Fuente: elaborada por las autoras

La mayoría de los proyectos normativos se ajustan a dos de estas categorías (normas de estructura y contenido de datos). Los estándares internacionales son considerados de estructura pues su objetivo fue regular los datos y no los continentes. Varios de los estándares normalizan la salida de datos en pantalla o en instrumentos impresos; a través de modelos detallados o reglas específicas (organización, redacción, ortografía) normalizan la formalización y la presentación de la información en los instrumentos de descripción. MAD3 y DACS son los únicos estándares que regulan cómo debe codificarse la información almacenada en entornos digitales y para ello proponen las normas de codificación que deben ser utilizadas.

Los procesos de normalización de la descripción prestaron atención, también, al control de los puntos de acceso. Los puntos de acceso constituyen un elemento esencial en la búsqueda y la recuperación de información en los sistemas de descripción archivística. Con el surgimiento de normas para la elaboración de representaciones de documentos y registros de autoridades archivísticas se hacía imprescindible contar, igualmente, con reglas 
para normalizar los puntos de acceso vinculados a nombres de instituciones, personas y familias y, por consiguiente, de lugares geográficos y materias. La identificación, recuperación y acceso de la información archivística va a depender, en gran medida, de la asignación adecuada de puntos de acceso en las descripciones.

Los archiveros comprendieron que para lograr una representación con la calidad exigida en los nuevos contextos era necesario normalizar los puntos de acceso de las descripciones. Como consecuencia, y tomando como base las normas bibliotecarias, algunos países han publicado reglas que rigen la elaboración y selección de los puntos de acceso en los sistemas de archivos.

En 1997, el National Council of Archives de Gran Bretaña publica las Rules for the Construction of Personal, Place and Corporate Names (RCPPCN); estas reglas constituyen una de las primeras normativas publicada en el área sobre el tema. En 2010, el Ministerio de Cultura de España publica la Norma para la Elaboración de Puntos de Acceso Normalizados de Nombres de Instituciones, Personas, Familias, Lugares y Materias en el Sistema de Descripción Archivistica de los Archivos Estatales (NEPAN). Las Rules for Archival Description de Canadá, las Orientaciones de Descripción Archivística de Portugal y el Manual de Descripción Multinivel de Castilla y León incluyen una parte dedicada a la regulación de los puntos de acceso. La primera versión de la norma estadounidense (DACS) contaba con un capítulo dedicado a los puntos de acceso, la segunda versión de 2013 ya no contiene esta parte. Los archiveros de Estados Unidos alegaron que no era necesario este capítulo pues se podía trabajar con las normas bibliotecarias. Estas son las normas que existen, hasta el momento, en el contexto archivístico para la normalización de los puntos de acceso.

Las normas presentadas, hasta el momento, rigen el contenido intelectual de las descripciones, diseñan sus estructuras y se orientan en el texto y el formato de los instrumentos de descripción. Sin embargo, existía un conjunto de cuestiones que estos estándares no podían resolver: ¿cómo interrelacionar la información de todas las entidades archivísticas? ¿Cómo facilitar la navegación por una arquitectura de información y recuperar la información específica? Además de crear normas de datos, contenidos y presentación, la comunidad archivística precisaba desarrollar estándares que tradujeran las normas del lenguaje natural al lenguaje propio de los entornos electrónicos. La computadora no puede procesar los datos representados en las descripciones, para ello, es necesario colocarlos en un idioma legible para la máquina, sólo así se pueden identificar y compartir los datos de un registro descriptivo.

Los profesionales necesitaban crear normas de codificación que convirtieran las estructuras de datos de los actuales estándares a un idioma entendible 
en el entorno electrónico. En las fases tempranas del desarrollo de un proyecto de lenguaje de codificación para archivos se consideró usar el Machine Readable Cataloging (MARC) como la base de las normas, puesto que es un estándar de marca muy conocido en el mundo bibliotecario y muchas instituciones lo usan para codificar instrumentos de descripción; pero, finalmente, se consideró que no era el mejor esquema disponible por varias razones. MARC era inadecuado porque los registros tenían una longitud máxima de pocos caracteres y presentaba dificultad para representar la información estructurada jerárquicamente debido a su limitado hospedaje.

El primer estándar archivístico creado y publicado como estructura de datos para facilitar la distribución en Internet de información detallada sobre fondos archivísticos a través de los instrumento de descripción fue el Encoded Archival Description (EAD). EAD refleja la estructura lógica y jerárquica de un instrumento de descripción de archivo y es compatible con la norma internacional ISAD $(G)$. Este estándar posibilita la difusión, acceso y navegabilidad, a través de la tecnología de redes, de la información descriptiva de las instituciones archivísticas.

Otro estándar de codificación creado y publicado para el contexto archivístico es el Encoded Archival Context-Corporate Bodies, Persons And Families (EAC-CPF). El esfuerzo por desarrollar EAC no es un hecho aislado. Estuvo muy vinculado con las actividades e iniciativas que desarrollaba la comunidad internacional de archivos en cuestiones de normalización. La norma ISAAR(CPF) fue el punto de partida para la creación de EAC, que sería un complemento de EAD, tal como ISAAR(CPF) lo es de ISAD(G). EAC es una norma para la gestión electrónica de la información de autoridades archivísticas y su contexto de producción. En la actualidad, el modelo descriptivo basado en la contextualización de los documentos (CIA, 2016), facilita el e-descubrimiento del recurso en la Web.

En los tiempos actuales, la representación de las entidades archivísticas debe realizarse respetando las pautas internacionales y nacionales recogidas en los estándares publicados. Resulta impensable representar información sin hacer uso de los metadatos y los contenidos establecidos en las regulaciones, máxime al considerar el paradigma en el que nos ubicamos, donde se prima facilitar la información con la finalidad de animar, mediante el fomento de una comunidad de usuarios participativa, a implicarse en el uso y conformación de la memoria patrimonial. 


\section{NORMALIZAR PARA FACILITAR LA CONTINUIDAD Y PRESERVACIÓN DE} RECURSOS DIGITALES

En la sociedad de la información y el conocimiento, la capacidad de los dispositivos procesadores y de almacenamiento de la información digital es elevada. Las leyes de Moore (capacidad exponencial de procesamiento y transmisión de la información y costes) y de Kryden (capacidad de almacenamiento y reducción del tamaño de los contenedores) nos permiten comprender la explosión experimentada en la producción de recursos digitales (volumen y variedad), así como los avances en su almacenamiento mediante la reducción de costes y tamaños de los dispositivos. La sociedad red genera abundancia de recursos digitales que precisan ser gestionados para potenciar y favorecer la producción, en términos de mantenimiento, reutilización, seguridad, acceso y que, finalmente, necesitan ser conservados de modo "disponible y accesible" a corto, medio y largo plazo con la finalidad de constituir patrimonio digital. Tanto el Informe sobre el Universo Digital (Dell-EMC, 2014), donde se demuestra el crecimiento de dicho universo y sus principales tendencias, como la Declaración de Vancouver sobre el patrimonio digital (Unesco, UBC, 2012) son reflejo fidedigno de esta explosión así como de la preocupación que sobre este patrimonio digital sostienen estudiosos, profesionales e instituciones. El ejercicio de preservar los recursos digitales debe contemplarse desde una doble temporalidad y finalidad: se preserva en el presente para reutilizar los datos e información, aunque también con el objetivo de asegurar su futuro. Preservar supone la conciliación entre la gestión del recurso digital (continuidad digital: corto y medio plazo) y su conservación (patrimonio digital: a largo plazo). El preservador debe asumir y coordinar los siguientes saberes: conocimientos sobre conservación física de recursos analógicos, conocimientos sobre amenazas y seguridad informática y de la información para identificar y gestionar riesgos a los que los recursos están sometidos; conocimiento de tendencias y avances para la conservación de los recursos -a corto, medio y largo plazo si este caso es posible- y conocimiento sobre modos y posibilidades de acceso, así como sobre las posibles y cambiantes necesidades de las comunidades específicas (usuarias presentes y futuras de los recursos). Pero además, el preservador debe ser un administrador de su unidad y ante todo un buen coordinador y gestor de estrategias para asegurar la implementación de políticas preservadoras, la colaboración con otros centros -resulta difícil asumir la preservación del patrimonio sin colaboración- y la obtención de recursos suficientes para su ejercicio preservador. 
Ante la variedad, volumen, vulnerabilidad y volatilidad de los recursos digitales, así como ante los requisitos de veracidad que se les exige en buena parte de los casos, han surgido un conjunto de normas orientadas a facilitar las estrategias empleadas para su preservación, destacando aquellas vinculadas a la interoperabilidad, almacenamiento y conservación de los recursos en repositorios, a la gestión de los riesgos a los que por sus características están expuestos y a la seguridad de la información, entre otras. Todas ellas son objeto de identificación en este enunciado bajo las siguientes categorías: normas facilitadoras de estrategias, normas centradas en los repositorios, normas destinadas a construir continuidad digital, normas vinculadas a la gestión del riesgo, normas orientadas a la seguridad de la información. A continuación se describen sucintamente.

\section{Normas facilitadoras de estrategias de preservación}

Dado que la volatilidad de los recursos digitales y su variedad dificultan la conservación de los mismos, así como su interoperabilidad, resulta fundamental la regulación de su estructura (los formatos digitales). Cada país debería de disponer de un inventario o catálogo oficial -o normalizado- actualizado de formatos. ${ }^{1}$

En esta línea, y con el objetivo de facilitar la interoperabilidad, en el 2012 se editó la norma ISO 13008 (ISO, 2012b), donde se detallan los requisitos de preservación específicos antes y durante el proceso, además de aquellos de tipo técnico y los procedimientos de migración y conversión (incluidos su programa y plan de actuación ante la planificación y el desempeño de la estrategia). Dicho estándar facilita la planificación de los recursos, la identificación de los requisitos y proporciona los procedimientos necesarios para llevar a cabo las estrategias de conversión y de migración. No se trata de un código elaborado para efectuar digitalización de masas documentales históricas, sino que se orienta a proporcionar continuidad digital en los entornos administrativos de las organizaciones.

El estándar es vital para normalizar los procesos de conversión y migración a pesar de no incorporar especificaciones para sistemas de respaldo (backup), de preservación de documentos digitales, de repositorios digitales y de conversión de formatos de documentos analógicos a digitales. En un plano nacional, muchos países disponen de regulaciones legislativas y normativas de carácter técnico facilitadoras de la interoperabilidad sobre los 
procedimientos de copiado auténtico, conversión y migración de ficheros digitales, como es el caso de la e-administración española. ${ }^{2}$ En relación a los formatos de preservación a largo plazo, existen diferentes normas ISO que regulan determinados formatos de documentos revisables o no revisables ISO 19005-1 (2005b), Norma Técnica de Interoperabilidad específica para estándares (España. Ministerio de Hacienda y Administraciones Públicas, 2012); ISO 32000-1 (2008a), tales como los formatos PDFA-1, válido para digitalizar documentos de recursos del Office, PDFA-2, garante de la legibilidad de documentos digitalizados como imágenes, y PDFA-3, con potencial para la captura de varios formatos de ficheros de diferente procedencia y contenido.

En relación a estrategias vinculadas a la interoperabilidad y continuidad digital destacamos, igualmente, una norma interesante para la digitalización de masas documentales no históricas, esto es, la norma ISO/TR 13028 (ISO, 2010c). Se trata de un informe técnico en el que se acuerdan directrices para la implementación de la digitalización de documentos destinadas a aquellas instituciones que experimenten procesos de digitalización en oficina de documentos originales en sus actividades diarias o bien de masas documentales no digitalizadas (digitalización retrospectiva no histórica) y que precisan ser digitalizadas para la continuidad de negocio. La norma establece pautas orientadas al logro de la veracidad del proceso, desde el enfoque legal y considerando los requisitos de evidencia del recurso, y dirigidas a fomentar la reutilización y accesibilidad del objeto de datos digitalizado, así como a establecer un procedimiento y los procesos que aseguren recursos digitales aptos para la conversión a largo plazo. Asimismo, incluye recomendaciones para la gestión de aquellos recursos originales no digitales tras su digitalización.

El empleo de metadatos para la comunidad archivística significa disponer de una estrategia de continuidad y de preservación del recurso digital. Además de las categorías de metadatos de carácter descriptivo e identificativo, características de todo recurso digital (metadatos básicos Dublin Core), en el archivo y en los sistemas de gestión para los documentos desplegados en las organizaciones, dada su singularidad y requisitos de evidencia documental, se precisa el uso de otras categorías funcionales y técnicas de metadatos de utilidad para comprender el contexto tecnológico en el que los objetos digitales (files) son creados y administrados, el contexto productivo y organizativo, sus necesidades de uso y reutilización -trayectoria pasada/futura-, así como otros elementos representativos que aseguren su preservación a corto,

2 España aprobó y publicó un conjunto de procedimientos de copiado auténtico y conversión entre documentos electrónicos (así como una guía explicativa) en el año 2011. 
medio y largo plazo. El objeto de datos más la información de contenido se conforman como paquete de información, de ahí la importancia del set de metadatos que se le atribuye y de sus especificaciones para realizarlo de modo sistematizado.

Las especificaciones de metadatos, por lo tanto, sirven para fraguar las representaciones de los recursos digitales que en este apartado citamos, únicamente, desde el enfoque preservador, dado que en este campo existen numerosas normas que son empleadas, tanto internacionalmente (serie de normas ISO 23081 o DoD-Interpares, por ejemplo) como en ámbitos nacionales (e-EMGDE para España, por ejemplo). Buena parte de las especificaciones de metadatos contienen categorías de metadatos vinculadas a la preservación. En este sentido, cada paquete de información que es capturado en un e-depósito incluye información de contenido -metadatos que representan su "contenido" - de naturaleza administrativa, estructural, identificativa o/y técnica y cuya utilidad asegura en la oficina productora la disposición para acceder y utilizar el recurso. Cada paquete de información creado/capturado y que es calificado de archivo - esto es, que forma parte de un sistema de gestión de documentos controlado archivísticamente- precisa de nuevos metadatos que den fe de su trayectoria, de su identidad contextual y que posibiliten su preservación como recurso, disponible, accesible y utilizable en el modo acordado a medio y largo plazo, asegurando su valor de evidencia. Esto hace referencia al conjunto de metadatos que aportan "[... ] la Información que un repositorio de preservación utiliza para asegurar los procesos de preservación digital” (PREMIS, 2008: 213) o la recogida del modelo OAIS, entendida como un conjunto de datos que conforman "[...] la información de contenido y que puede ser descrita [en términos de:] Procedencia, Referencia, Fijeza, Contexto e Información de los Derechos de Acceso"3 (ISO, 2012c: 24). Desde este enfoque, destacamos tres especificaciones de consideración: una norma destinada a pautar la encapsulación de los metadatos ante la transferencia de ficheros, especificación METS (Library of Congress, s. f.), una norma destinada a la gestión de los metadatos de preservación en los repositorios, especificación PREMIS, y una norma en la que se detalla un modelo de metadatos para un repositorio normalizado, como es el modelo de ficheros abiertos OAIS.

La especificación METS ha sido desarrollada por la Library of Congress para el envío de ficheros, de imágenes o cualquier objeto multimedia de una biblioteca o repositorio digital que emplea la estructura XML. Codifica los metadatos para la gestión de los objetos digitales y facilita su transferencia de 
repositorio en repositorio, encapsulando los metadatos mismos del recurso y proceso. Codifica y representa metadatos descriptivos, técnicos, de derechos, administrativos y estructurales (mapa estructural) en relación con los objetos digitales y se expresa en XML. Su empleo se ve incrementado porque facilita la integración de colecciones. PREMIS es editada en su primera versión en el 2005 (diccionario de datos) y surge mediante una investigación colaborativa de un panel de más de 30 expertos de cinco países, constituido como grupo de trabajo en el 2003, para la identificación de estrategias para la implementación de metadatos en e-depósitos. El proyecto fue financiado por OCLC+RLG. La última versión, 3.0, ha sido editada en el 2015. Es uno de los estándares por excelencia de trabajo en metadatos de preservación, mantenido por la Library of Congress, que se presenta como esquema XML y que constituye un catálogo de metadatos de preservación específicos. Es un modelo conceptual para la implementación de metadatos de preservación para cualquier repositorio de una unidad de información. Se utiliza, especialmente, en el diseño de repositorios, así como para su evaluación y para el intercambio de paquetes de información archivada entre e-depósitos. En la norma se especifican las entidades (tipo de recurso, objeto de datos y condiciones técnicas, agentes que intervienen en su concreción, evento que lo justifica o genera, derechos y permisos a los que está sujeto), subentidades (tipos de objetos, de permisos y derechos, de persona que interviene, etc.), atributos, relaciones (estructurales, de dependencia, de derivación) y unidades semánticas (ejemplo, el formato de un objeto, el tipo en un agente, el tipo de permiso en la entidad derechos). De este modo, cualquier paquete de información de contenido aporta información sobre su presentación -documento, expediente, libro, estructura capitular, estructuras de formatos en los que muestra una apariencia, etc. -, sobre sus características técnicas de creación y conversión/migración, sobre las circunstancias que justifican su creación, sobre el productor-creador del mismo, sobre los derechos de propiedad intelectual a los que está sujeto o permisos de acceso y uso, sobre aspectos vinculados a su funcionalidad y trayectoria futura para la preservación, etc. PREMIS no es una especificación autónoma y debe implementarse en un repositorio. Se coordina de modo eficiente con METS. El estándar METS garantiza el envío/recepción al/en el e-depósito del recurso digital y el paquete de información asociado a él en las condiciones de representación acordadas. En el e-depósito, PREMIS controla y completa el conjunto de metadatos necesarios para gestionar el recurso -así como sus paquetes de información sobre el contenido- y el modo en que es almacenado para su futura disposición. La especificación OAIS se explica con detalle en la siguiente categoría, dado su alcance en e-depósitos. 
Finalmente, para asegurar la preservación de la información electrónica a largo plazo cabe señalar, además, tres normas cuyas finalidades se orientan a ello. La primera es la norma ISO/TR 15801:2009 (ISO, 2009b), la cual se centra en directrices para asegurar la integridad y veracidad de la información; la norma ISO 14641-1:2012 (ISO, 2012f) incluye disposiciones para asegurar la información electrónica en un sistema de información y resultan de gran interés tanto los requisitos a considerar para los soportes como los apartados finales donde se instruye para conformar contratos y convenios con terceras partes. A su vez, desde el 2005 se dispone del informe técnico ISO/TR 18492:2005 (ISO, 2005a), donde se establecen pautas para asegurar la conservación a largo plazo de los documentos.

\section{Normas centradas en repositorios digitales}

La longevidad digital de los recursos electrónicos, nos recuerdan Horsman y Spork (2013: 14), incluye los requisitos de accesibilidad, legibilidad y comprensibilidad, precisando de e-depósitos considerados como herramientas básicas. Un repositorio seguro debe cumplir los requisitos que establecen las normas técnicas internacionales para la auditoría y certificación de su fiabilidad y se conforma como una plataforma digital de conservación (España. Ministerio de Industria, Energía y Turismo, 2015: 4). En materia de repositorio con ficheros abiertos se destaca la norma ISO-14721 (ISO, 2012c) ${ }^{4} \mathrm{cu}-$ ya primera versión data del 2003. Se trata de una norma básica a considerar para comprender la dinámica, estructura y administración de un e-depósito. Este documento ha sido aprobado para su publicación por el Consejo de Administración del Comité Consultivo para los Sistemas de Datos Espaciales (CCSDS) y representa el acuerdo técnico consensuado de las agencias miembros del CCSDS participantes en el seno del Comité Técnico ISO/TC20 Aeronaves y vehículos especiales y su Subcomité SC13, Sistemas de transferencia de información y datos espaciales.

Esta norma representa un modelo de referencia para un sistema abierto de información de archivo (OAIS). Esto es, definido como "[...] Archivo, que una organización opera, y que puede formar parte de una organización más amplia, de personas y sistemas, que acepta la responsabilidad de conservar información y mantenerla disponible para una Comunidad Específica" (Norma Española, 2015c: 5). Se trata de un modelo basado en el reconocimiento de entidades funcionales que incluyen desde el proceso de captura hasta el de su consulta.

4 Acceso al modelo en http://public.ccsds.org/publications/archive/650x0m2.pdf. En SC1-España del CTN50 de Aenor se ha traducido y editado esta práctica recomendada, como norma española. 
La norma define una serie de requisitos que facilitan la normalización del proceso de conservación de los objetos de datos y de su información representativa a largo plazo. Igualmente, especifica requisitos cuyo cumplimiento e implementación favorecen su acceso. En este modelo de referencia se otorga una atención especial a la información digital, tanto como una forma inicial de información almacenada o/y como una información de apoyo para materiales archivados físicos y digitales. Puede ser aplicado a cualquier archivo que disponga de información a largo plazo y es importante para la comparación y diseño de herramientas que gestionen la información sobre una base temporal y a largo plazo. Ante todo no especifica un diseño o una implantación pero sí es útil para su desarrollo normalizado. Para la función certificadora de repositorios se dispone de una serie de normas que los archiveros deben de conocer y aplicar:

- Norma de certificación por tercera parte (denominada en el contexto de la evaluación como "evaluación de segunda parte"): informe técnico ISO/TR 17068:2012 (ISO, 2012e). El estándar facilita que el cliente que ha contratado un servicio de gestión de repositorio tenga cierta seguridad sobre cómo se realiza dicha gestión. Esto es, sobre garantías en torno a los servicios, personas y sistemas que se encargan de asegurar los recursos digitales. Por ello, es una norma verificadora por "tercera parte" de los repositorios, aunque a demanda del cliente.

- Norma de certificación por entidades certificadoras reconocidas. El canon ISO/16363:2012 (ISO, 2012d) permite asegurar el trabajo para comprobar los requisitos de un repositorio digital. Incluye requisitos que deben considerarse para una correcta verificación del funcionamiento de los repositorios (cuyo incumplimiento implica riesgos y no conformidades). La norma es un producto basado en una herramienta pensada para la certificación de e-depósitos conocida como modelo TRAC, cuya primera versión data del 2007. Establece una línea básica de evaluación centrada en un repositorio digital de confianza mediante listas de comparación. Es el modelo de referencia para la construcción de la versión OAIS del 2012.

- Norma de reconocimiento de las entidades certificadoras de repositorios. La norma ISO 16919 (ISO, 2014b) es un producto específico en el que se regula el procedimiento certificador y las cualidades y requisitos a cumplir por las entidades que certifican repositorios. Esta norma está basada en la norma ISO 17021-1 (ISO, 2015a) sobre normalización de requisitos para certificar un sistema normalizado aplicable a cualquier sistema y sobre una especificación pensada para repositorios. 
Además de la certificación o evaluación de los e-depósitos empleando las antedichas normas, su verificación del riesgo se efectúa mediante el uso de aplicaciones específicas, como son, entre otras, la herramienta de autoevaluación DRAMBORA, ${ }^{5}$ la aplicación DAF-Data-Audit ${ }^{6}$ o el Proyecto NESTOR, convertido en norma nacional alemana. ${ }^{7}$

\section{Normas de apoyo a la continuidad digital}

Entendemos por continuidad del negocio la capacidad estratégica y táctica que toda organización dispone para planificar y responder ante incidencias e interrupciones del negocio con la finalidad de que la operatividad del negocio continúe. Se entiende que previamente ha sido definido un nivel de riesgo aceptable. Hablamos de cierta capacidad para identificar las amenazas, definir los riesgos, conocer su impacto y acordar su grado o nivel de aceptación en atención a los recursos disponibles en la organización.

Sobre continuidad del negocio han existido y existen diversas normas UNE que son traducción de una norma británica (BS 25999 Part 1 and 2) convertida en norma ISO (ISO 22301, 2012g). Esta norma ISO, revisada con data del 2014, ha sido traducida y aceptada en España como norma UNE-EN-ISO 22301 (Norma Española, 2015a). ${ }^{8}$ Dichos códigos están estrechamente relacionados con los riesgos pues son una alternativa a actuaciones cuando se producen incidentes e interrupciones. Complementan las políticas de gestión de riesgo dado que se orientan a hacer factible la continuidad del negocio. Sobre esta base se han desarrollado modelos para entornos digitales y ha surgido el concepto de continuidad digital (tan adecuadamente trabajado y aplicado desde los Archivos Nacionales de UK y de Australia). La reciente traducción

5 La herramienta DRAMBORA fue creada por el Centro de Preservación Europeo y de modo conjunto junto con ISO/tc46/sc11 en 2009. Disponible en http://www.drambora.org.

6 Esta herramienta fue creada en el 2006 por el Humanities Advanced Technology and Information Institut (HATII) de la Universidad de Glasgow junto con el DCC. Su objetivo ha sido el de asegurar los recursos científicos de la Universidad en el repositorio institucional. Disponible en http://www.data-audit.eu.

7 El proyecto NESTOR genera en el 2008 un catálogo de criterios para verificar repositorios de confianza. Se basa en estándares nacionales alemanes y en otros estándares como puede ser TRAC. Esta aplicación se está trabajando como norma DIN 31644. Disponible en http://www. din.de.

8 Señalamos la existencia de dos partes diferenciadas y editadas como normas separadas desde el 2010. La norma UNE 71599-1: 2010 (BS 25999 Part 1) en la que se especifica el modelo fundamentado de la gestión de la continuidad del negocio, siguiendo el ciclo de gestión PDCA y la parte 2, UNE 71599-2 (2010) (BS 25999 Part 2), en la que se detallan las especificaciones. En el presente, el modelo ISO 22301 (2014) ha representado la fuente de traducción Aenor que fue editada en el 2015. La norma UNE-ISO es promovida por el CTN 196 de protección y seguridad de los Ciudadanos. Véase también Norma Española UNE-ISO española (Norma Española, 2015b). 
de las normas UNE-ISO 22301 (Norma Española, 2015a) e UNE-ISO 22302 (2015) (Norma Española, 2015b) incluye un conjunto de especificaciones de requisitos y directrices para un sistema de gestión de la continuidad de negocio, encargado de proteger a la organización de todo tipo de incidencias que provoquen una interrupción de las actividades, además de gestionar el riesgo y garantizar su recuperación en caso de que acontezca. En los entornos tecnológicos, así como en los marcos MSS se prima la gestión del riesgo. A continuación detallamos normas que regulan su fundamentación, metodología, sistema de gestión y certificación.

\section{Normas para la gestión y certificación del riesgo en las entidades}

Lemieux (2004) describe el riesgo como el evento o acción que no ha ocurrido todavía y que, teniendo una cierta probabilidad de ocurrencia, implica una consecuencia e impacto en caso de acontecer. Su condición potencial lo caracteriza como un concepto relativo vinculado a una toma de decisiones y a la programación y ejecución de acciones derivadas de dicha toma sobre el futuro en un contexto organizacional.

En el entorno de gestión de documentos electrónicos el riesgo es una constante, dada la vulnerabilidad del recurso digital. La comunidad de archiveros ha desarrollado un informe técnico para apreciar áreas de riesgo y amenazas en un sistema de gestión documental, así como para pautar un procedimiento de análisis del riesgo. Nos referimos al informe técnico ISO/ TR 18128 (2014d) de aplicación en cualquier organización. Se trata de una metodología de trabajo muy recomendada para el análisis y la gestión del riesgo para el sistema de gestión para los documentos, en el marco normalizado de gestión (MSS) de ISO 30300 (ISO, 2011c).

La gestión del riesgo puede (y debería) gestionarse y debe entenderse como un proceso sistemático de un conjunto de acciones necesarias para identificar y controlar todos los riesgos, de naturaleza "proactiva" (encabezando riesgos) y que debe emplearse como herramienta básica ante cualquier toma de decisiones para la entidad y, muy especialmente, a considerar ante los entornos electrónicos. Sin lugar a dudas, debe orientarse a prevenir pérdidas y capitalizar oportunidades para implementar las actividades de la organización.

En lo referente a la identificación y gestión de riesgos resulta muy conveniente conocer y aplicar las normas del marco MSS serie ISO 31000. Se hace referencia a la serie normativa sobre la administración normalizada de un sistema de gestión de riesgos en una organización. Se trata de un modelo para 
lograr y certificar sistemas normalizados de gestión del riesgo. Destacamos, a modo de iniciación a su conceptualización, la Guía 73 (Norma Española, 2010) en la que se establece el vocabulario básico. La norma donde se fundamenta la identificación y gestión del riesgo es la ISO 31000 (ISO, 2009c). En ella se explica la metodología de trabajo y se argumenta el modelo de un sistema de gestión del riesgo.

La norma UNE-ISO 31010 (Norma Española, 2013a) explica y detalla las técnicas empleadas para la identificación y análisis de los riesgos. Destacamos el contenido específico de esta última norma, destinado a presentar las técnicas de "apreciación" del riesgo, así como sus anexos A y B, donde se establecen una relación comparada de técnicas de apreciación del riesgo y su detalle descriptivo, para aportar al lector suficiente información operativa o/y en su caso informativa para animar a su aplicación. Estrechamente ligado al riesgo vinculamos el concepto de (in)seguridad de la información.

Los procedimientos para evitarla han sido normalizados mediante la serie de normas que se presentan en la categoría siguiente.

\section{Normativa vinculada a construir y certificar seguridad de la información}

La vulnerabilidad del recurso digital se ve mediatizada igualmente por los esfuerzos de asegurar su integridad y autenticidad, así como de controlar la seguridad de la información contenida en el mismo. A este respecto, recordamos que sucesivos informes sobre el universo digital describen cómo buena parte de los datos integrantes del mismo carecen de seguridad (hasta un $45 \%$ en el informe anteriormente citado del 2014). En esta misma línea, algunos estudios sobre los datos en la nube indican prácticas de las empresas gestoras y almacenadoras de datos en las que los criterios de seguridad y, por lo tanto, la seguridad de los datos, su accesibilidad, no puede ser comprobada o verificada, siendo percibidas como riesgos por las organizaciones. En cada nación, el Estado o las entidades jurídicas de alcance nacional, federal, autonómico o local deberían acordar un esquema nacional de seguridad y desarrollar guías y directrices que permitieran construir seguridad en sus administraciones electrónicas, en el supuesto de que no lo hayan realizado. En el caso de España, se dispone de un amplio entramado de guías, fuentes y disposiciones que facilitan la seguridad de la información. Este entramado se regula con un alcance general y se detalla y articula en directrices específicas 
a determinados contenidos. ${ }^{9}$ Por ejemplo, para la seguridad de los datos en la nube se establece una guía de actuación. ${ }^{10}$

El Esquema Nacional de Seguridad (ENS) ${ }^{11}$ aplicado en la e-administración española se sustenta sobre la fundamentación ISO existente en materia de seguridad de la información. La seguridad de la información en ISO se articula como un marco MSS orientado a la administración normalizada de la gestión de un sistema de seguridad de la información. Al igual que para la gestión de la continuidad digital, su origen se remonta a normativa de seguridad británica. El modelo normalizado se reconoce bajo la serie ISO 27000 (ISO, 2014a). La norma de controles de seguridad de la información es la ISO 27001 (ISO, 2013). Esta serie de normas se encuentra muy desarrollada. Se dispone de normas de directrices (Norma Española, 2013b; ISO 27003, 2010d), de normas de evaluación (ISO 27004, 2016b) y de certificación (ISO 27006, 2015c). Esta serie dispone de una norma específica complementaria para la gestión del riesgo en seguridad de la información: ISO 27005. Esta serie de estándares se plantea qué requiere ser protegido, razones, enemigos y métodos de cómo proteger. El modelo ISO 27000 se fundamenta sobre tres pilares conocidos, vinculados al valor de evidencia de los recursos digitales: confidencialidad, asegurando que sólo quienes estén autorizados pueden acceder a la información; integridad, asegurando que la información y sus métodos de proceso son exactos y completos y, finalmente, disponibilidad, asegurando que los usuarios autorizados tienen acceso a la información cuando lo requieren. Entendemos que dichos pilares son suficientemente explicativos para que el archivero conozca y emplee la normativa ISO.

\section{NORMALIZAR PARA CONSTRUIR Y CERTIFICAR SISTEMAS DE CALIDAD} Y EXCELENCIA EMPRESARIAL

El conjunto de modelos normalizados de gestión de sistemas del riesgo, de la seguridad, de la información, así como de sistemas de gestión para los documentos es conocido bajo el marco MSS de ISO. Entre todos ellos destaca por

9 Acceso a todas las guías editadas: https://www.ccn-cert.cni.es/index.php?option=com_wrapper\&view $=$ wrapper $\&$ Itemid $=188 \&$ lang $=e$

10 Guía/norma de seguridad de las TIC (CCN-STIC-823). Seguridad en entornos Cloud. Disponible en https://www.ccn-cert.cni.es/publico/seriesCCN-STIC/series/800-Esquema_Nacional_de_Seguridad/823-Seguridad-en-entornos-cloud/823-Cloud_Computing_ENS.pdf

11 El ENS está constituido por los principios básicos y requisitos mínimos requeridos para una protección adecuada de la información. Se aplica en la e-administración para asegurar el acceso, integridad, disponibilidad, autenticidad, confidencialidad, trazabilidad y conservación de los datos, informaciones y servicios utilizados en medios electrónicos que gestionen en el ejercicio de sus competencias. 
su alcance y expansión mundial el de la calidad y excelencia empresarial, esto es, el normalizado bajo la serie de normas conocidas como ISO 9000 (ISO, 2015d). La última revisión de la norma adopta un nuevo marco de referencia en cuanto a su estructura y terminología de normalización de los contenidos de los MSS, cuyo primer exponente elaborado bajo dichos acuerdos en el seno del Grupo de Trabajo de Normalización de Estándares de ISO fue, curiosamente, la norma ISO 30300 (ISO, 2011c). Desde el enfoque archivístico señalamos con satisfacción que el código ISO 9000 (ISO, 2015d) incluye e integra los conceptos de la norma ISO 303011 en lo referente a gestión de documentos. El modelo de diseño, implementación y evaluación de los sistemas de gestión para los documentos en las entidades es normalizado bajo la serie ISO 30300 (ISO, 2011c). Destacamos sus normas de desarrollo presentes para fundamentos y vocabulario (ISO 30300, ISO, 2011c), la norma de requisitos de gestión ISO 30301 (ISO, 2011d) y la guía para su implementación, ISO 30302 (ISO, 2015b). A su vez, se han trazado futuras normas aún en desarrollo que completan el modelo MSS, como los proyectos de normas destinados a la evaluación y certificación. Igualmente, deben ser considerados otros códigos satélites y complementarios, tales como ISO 15489 Partes 1 (ISO, 2016a) y 2 (ISO, 2001); ISO 26122 (ISO, 2008b); ISO 16175 Partes 1 a 3 (ISO, 2010a, 2011b, 2010b); ISO 23081, Partes 1 a 3 (ISO, 2006, 2009a, 2011a). El primero de los códigos ISO 15489-1 es una actualización de una versión anterior del 2001, aunque incluye importantes modificaciones (ISO, 2016a) en fundamentos y vocabulario; por razones de espacio y objetivos de trabajo sus contenidos no son tratados en este ensayo. Este conjunto de estándares responde a las necesidades de construir, implementar y certificar calidad y excelencia empresarial. Buena parte de los archivos son entendidos como unidades y centros de información cuya administración está sujeta a rendición de cuentas, en las que se incluye un interés por demostrar gestión de calidad y excelencia. Smit (2013) señala para el archivero un perfil de gestor de la calidad entre el conjunto de cambios que el profesional de la gestión de documentos está asumiendo (o debe asumir). Su metamorfosis como auditor y gestor de calidad es una experiencia en múltiples entidades para lo relativo a los procesos de soporte de información y comunicación en toda organización, aunque también para sostenimiento y gestión documental necesarios para la construcción, implementación, verificación y certificación de la calidad organizacional. Marín Agudelo (2012: 89) expone que entre la gestión documental y la calidad total existen amplias relaciones orientadas a la auditoría de la información con la idea de evaluación de información en las organizaciones y con la finalidad de identificar no calidades y disfuncionalidades y reflexionar sobre posibles mejoras. 
En este sentido, la revisión de la serie ISO 9000 (ISO, 2015d) otorga una nueva vía para que el archivero pueda coordinar lo estipulado; al respecto, tanto en ISO 15489 (ISO, 2016a) como en el marco MSS ISO 30300 (ISO, 2011c), aunando gestión documental y gestión de calidad, aunque también considerando que su unidad puede demostrar calidad. En esta línea, las normas que el archivero interesado en ello debe conocer y dominar son las normas conformadas por la serie ISO 9000 y, específicamente, en lo tocante a fundamentación y terminología, la norma ISO 9000 (ISO, 2015d); en lo referente a requisitos, la norma ISO 9001 (ISO, 2015e); en lo referente a implementación sostenida ISO 9004 (Norma Española, 2009); en lo relacionado con evaluación, la norma ISO 19011 (ISO, 2012a) o UNE-ISO, 19011 (Norma Española, 2012). Finalmente, en lo vinculante con la certificación de cumplimiento de requisitos (ISO 9001) y éxito sostenido (ISO 9004), el código ISO 17021-1 (ISO, 2015a).

\section{Consideraciones finales}

Los nuevos contextos sociales, económicos, políticos y culturales exigen que los profesionales de información tengan un amplio conocimiento y dominio de los estándares pues sólo así conquistarán el rol operativo y estratégico que la sociedad exige. La interdisciplinariedad inherente a la aplicación de las normas (reguladoras de áreas tan diversas como calidad, riesgos, seguridad, tecnologías, gestión, información) precisa un reflejo que se incluya en los planes de estudio. En esta línea, se expresa Ortiz-Repiso (2015) al defender la necesidad de actualizar los planes de estudio y considerar reforzar la interdisciplinariedad, además de destacar los perfiles de arquitecto y preservador de la información, perfiles en los que el empleo de los estándares presentados se torna fundamental.

Se ha puesto de relieve cómo la presencia incondicional de las tecnologías en los sistemas de archivos, el surgimiento de nuevos usuarios y la globalización de la información exigen mudar las concepciones clásicas en lo referente a la descripción, la búsqueda y el acceso a la información. En esta línea, Alvite Díez (2012: 182) expone que tradicionalmente el catálogo fue la herramienta de búsqueda y acceso más utilizada, pero internet y los continuos avances tecnológicos permitieron la disponibilidad de catálogos online que trajeron consigo transformaciones en la recuperación y el acceso a cualquier recurso de información a través del uso de interfaces cada día más perfeccionadas.

Los profesionales de la información, en especial los archiveros, tienen que asumir estos nuevos retos y, para ello, deben adoptar los modelos actuales de 
representación y recuperación de recursos. El uso normalizado de las estructuras de datos, contenidos y codificación para crear sistemas integrados de descripción facilita y hace factible la recuperación, el acceso y el intercambio de información entre usuarios procedentes de diferentes realidades.

Por otro lado, las normas facilitadoras de la continuidad a corto y medio plazo y de la preservación de los recursos a largo plazo responden a estrategias diversas de actuación: normalización de formatos y de soportes, migración, conversión, digitalización, empleo de metadatos, sistematización y verificación de repositorios de confianza y control de acciones para conservar documentos e información a largo plazo, así como para asegurar dicha información, trabajando seguridad, continuidad de negocio y gestión del riesgo. De igual modo, se han destacado estándares para participar en la construcción de administraciones con sistemas normalizados MSS, así como para el diseño de sistemas documentales normalizados propiamente dichos que puedan ser certificados.

La sociedad moderna demanda profesionales de la información que sean capaces de actuar en contextos de trabajo diferentes. Tal y como Smit (2013) ha desvelado, los nuevos especialistas deben poseer las habilidades para implementar un conjunto de operaciones que engloba la gestión de la información, entendida como un recurso, como un producto y, en último término, como un valor o activo en la organización. Para todo ello, se debe modelar el perfil de su labor más allá del tradicional gestor de documentos, pues ante todo su ejercicio profesional se efectúa sobre un territorio más amplio que es delimitado por el contexto en el que se producen los documentos y las necesidades presentes y futuras de la comunidad específica para la que se gestionan y conservan. En el espacio hipotético entre producción contextualizada y necesidades de consulta y reutilización, los recursos digitales deben afrontarse de modo seguro, con veracidad, con calidad, con riesgos gestionados, administrados de modo sistemático y con mayor inteligencia y representados de manera que la comunidad específica pueda identificarlos y recuperarlos a lo largo del tiempo. En esta tesitura, el archivero debe aprender a cooperar con otros profesionales aunque, también, a fundamentar su trabajo en modelos normalizados y homogéneos que recojan todo tipo de prácticas.

¿Cómo lograr que los profesionales alcancen estos conocimientos y las habilidades necesarias para aplicar y trabajar con las nuevas regulaciones? Una respuesta parece indiscutible: insistir en la formación académica y profesional y en el desarrollo de investigaciones relacionadas con los estándares y sus aplicaciones es el camino correcto. En este sentido, Smit (2013) señala la diversidad de perfiles del archivero necesarios para aportar un adecuado servicio en la conocida "infoesfera" teorizada por Luciano Floridi, entre los 
que destacan auditor de documentos (sistemas de gestión para los documentos verificados), controlador de documentos (análisis de riesgos y control de sistemas documentales), gestor de la calidad y de la veracidad documental (creando, rediseñando y describiendo procesos y funciones, y sosteniendo la base documental de la calidad), arquitecto documental (arquitecto de políticas de la información, interoperabilidad y seguridad), gestor de contenidos (controlando la ingesta de ficheros, su almacenamiento y disposición para el acceso en e-depósitos), preservador de recursos digitales (planificando y gestionando en los repositorios la conservación y disponibilidad de los datos, documentos e información en el tiempo) y responsable de su e-descubrimiento (disponiendo datos, información y documentos para su acceso, uso y reutilización a corto, medio y largo plazo) .

Se hace imprescindible, entonces, concentrarse en la academia y los cursos universitarios. Los cursos de graduación y posgraduación en Ciencias de la Información, Documentación o Archivística deben considerar en sus programas de estudios disciplinas que incluyan contenidos relacionados con las normativas anteriormente señaladas. En este sentido de incorporar asignaturas específicas sobre normas, además de las clásicas vinculadas a la organización y representación de contenidos o a la preservación digital, algunas universidades así lo han realizado, ejemplo de ello son la Universidad de Salamanca (asignatura Estándares para Archivos Electrónicos, en Grado y de naturaleza optativa) y la Universidad Carlos III de Madrid (asignatura Sistemas de Gestión de Documentos. Normas Técnicas, en Máster y de naturaleza obligatoria). Las investigaciones en esta área del conocimiento manifiestan la necesidad de insistir en la inclusión de las normas como objeto de investigación, desde el enfoque de conocer su impacto, contribuir a su mejora y a la creación de otras tantas que faciliten el ejercicio de "archivar".

Sobre esta línea, Marín Agudelo (2012: 93) hace un llamado sobre la necesidad de actualizar programas de formación específicos de archivística que hagan frente a los escenarios tecnológicos y digitales así como a la necesidad de estudiar de modo más sistematizado las implicaciones de la administración de archivos y la gestión de calidad. Moreiro-González (2015), igualmente, observa a la economía digital como una oportunidad para revisar las titulaciones y adaptar los perfiles profesionales.

La introducción en los currículos de estas cuestiones puede contribuir al mejoramiento de las prácticas, al desarrollo de la archivística y a una mayor inserción de los archiveros en los mercados laborales. Es responsabilidad de profesores e investigadores abordar y discutir desde las salas de aulas temas relacionados con normas de gestión, metadatos, representación, recuperación, continuidad digital, preservación, calidad, entre otras, pues 
ante contextos de cambio, objeto, finalidad y responsabilidades permanecen en continuada metamorfosis.

\section{REFERENCIAS}

Alvite Díez, M. 2012. "Redefiniendo el catálogo. Expectativas de las interfaces de descubrimiento centradas en el usuario”. Investigación Bibliotecológica 26 (56): 181- 204.

Bonal, J. J. Generelo y C. Travesí. 2006. Manual de Descripción Multinivel: propuesta de adaptación de las normas internacionales de descripción archivística, 2a. ed. Valladolid: Consejería de Educación y Cultura. Fecha de consulta: 17 de julio de 2015. http://www.archivoscastillayleon.jcyl.es/web/jcyl/binarios/898/985/Manual\%20de \%20D escripci\%C3\%B3n\%20Multinivel_2006,1.pdf?blobheader=application $\% 2 \mathrm{Fpdf} \% 3 \mathrm{Bch}$ ars et $\% 3 \mathrm{DUTF}-8$ \&blobnocache $=$ true

Bureau Canadien des Archivistes. 2008. Rules for Archival Description. Ottawa: Bureau Canadien des Archivistes. Fecha de consulta: 30 de junio de 2015. http:// www.cdncouncilarchives.ca/archdesrules.html

Comisión de Normas Españolas de Descripción Archivística. 2012. Modelo conceptual de descripción archivística y requisitos de datos básicos de las descripciones de documentos de archivo, agentes y funciones. Fecha de consulta: 15 de julio de 2015. http://www.mcu.es/archivos/docs/NEDA_MCDA_P1_P2_20120618.pdf

CIA (Consejo Internacional de Archivos). 2000. ISAD (G): Norma Internacional para la Descripción Archivística. Madrid: Dirección General del Libro, Archivos y Bibliotecas, Subdirección General de los Archivos Estatales.

CIA. 2004. ISAAR (CPF): Norma Internacional sobre los Registros de Autoridad de Archivos Relativos a Instituciones, Personas y Familias, 2a. ed. Madrid: Dirección General del Libro, Archivos y Bibliotecas, Subdirección General de los Archivos Estatales.

CIA. 2008a. ISDF: Norma Internacional para la Descripción de funciones. Comité de Buenas Prácticas y Normas Profesionales del CIA. Madrid: Dirección General del Libro, Archivos y Bibliotecas, Subdirección General de los Archivos Estatales.

CIA. 2008b. ISIAH: Norma Internacional para las Instituciones que Custodian Recursos Archivísticos. Comité de Buenas Prácticas y Normas Profesionales del CIA. Madrid: Dirección General del Libro, Archivos y Bibliotecas, Subdirección General de los Archivos Estatales.

CIA. 2016. Records in Contexts. A conceptual model por archival description. V0.1. Fecha de consulta: 8 de febrero de 2018. https://www.ica.org/en/egad-ric-conceptual-model

Conselho Nacional de Arquivos. 2006. NOBRADE: Norma Brasileira de descrição arquivística. Rio de Janeiro: Conselho Nacional de Arquivos.

Cook, M. y M. Procter. 2000. Manual of Archival Description, 3a. ed. Aldershot: Gower.

Dell-EMC. Digital Universe Study. 2014. Discover the digital universe of opportunities: rich data and the increasing value of the internet of things. Fecha de consulta: 8 de febrero de 2018. https://www.emc.com/leadership/digital-universe/index.htm 
Direcção Geral de Arquivos. 2007. Orientações para a Descrição Arquivística, 2a. ed. Lisboa: Direcção Geral de Arquivos, Grupo de Trabalhode Normalização Descrição em Arquivo. Fecha de consulta: 17 de julio de 2015. http://www.aefp.org. es/NS/Documentos/NormasDescriptivas/oda1-2-3\%282vers.\%29.pdf

Encoded Archival Context (EAC). Fecha de consulta: 15 de julio de 2015. http://eac. staatsbibliothek-berlin.de/

Encoded Archival Description (EAD). Fecha de consulta: 15 de julio de 2015. http:// www.loc.gov/ead/ead2002a.html

España. Ministerio de Hacienda y Administraciones Públicas. 2012. Resolución de 3 de octubre de 2012 de la Secretaría de Estado de Administraciones Públicas por la que se aprueba la norma técnica de interoperabilidad de Catálogo de Estándares. BOE 262. 31 de octubre de 2012: 76713-76723. Fecha de consulta: 8 de febrero de 2018. https://www.boe.es/buscar/doc.php?id=BOE-A-2012-13501

España. Ministerio de Educación, Cultura y Deporte. Biblioteca Nacional. 2013. Borrador de proyecto de real decreto por el que se regula el depósito legal de las publicaciones electrónicas. Fecha de consulta: 17 de julio de 2015. http://www.mecd.gob. es/servicios-al-ciudadano-mecd/dms/mecd/servicios-al- ciudadano-mecd/participacion-publica/cerrados/2013/dl-publicaciones-electronicas/dl-publicaciones-electronicas-v1.pdf

España. Ministerio de Educación, Cultura y Deporte. Subdirección General de Archivos Estatales. 2005. Norma española de Descripción Archivística (NEDA). Madrid: Ministerio de Cultura, Subdirección General de Archivos Estatales.

España. Ministerio de Industria, Energía y Turismo. 2015. Libro Blanco para el diseño de titulaciones universitarias en el marco de la Economía Digital. Madrid: Cyan, proyectos y productos editoriales.

España. Ministerio de Industria, Energía y Turismo. 2010. Norma para la Elaboración de Puntos de Acceso Normalizados de Nombres de Instituciones, Personas, Familias, Lugares y Materias en el Sistema de Descripción Archivística de los Archivos Estatales. Madrid: Ministerio de Educación, Cultura y Deporte, Subdirección General de Archivos Estatales. Fecha de consulta: 30 de junio de 2015. http://www.mcu.es/ archivos/docs/Novedades/Norma_puntos_acceso2010.pdf

Estudio del Universo Digital de EMC con investigación y análisis por IDC. 2014. Fecha de consulta: 27 de abril de 2005. http://spain.emc.com/leadership/digital- universe/index.htm

Grupo de Trabajo de Autoridades de Aragón. 2014. ARANOR: Norma Aragonesa para la Descripción de Autoridades de Archivos, 2a. ed. Aragón: Gobierno de Aragón, Departamento de Educación, Cultura y Deporte. Fecha de consulta: 27 de junio de 2016. http://www.anabad.org/admin/archivo/docdow.php?id=506

Horsman, P. y R. Spork. 2013. "How do you build an e-depot? Lessons from the Rotterdam case". Comma 2: 43-58.

ISO (International Organization for Standardization). 2001. ISO/TR 15489-2:2001. Information and Documentation-Records management- Part 2. Guidelines. Ginebra. ISO. (Actualmente, en revision)

ISO. 2005a. ISO/TR 18492:2005. Long-term preservation of electronic document-based information. Ginebra: ISO.

ISO. 2005b. ISO. 19005-1. Document management -- Electronic document file format for long-term preservation -- Part 1: Use of PDF 1.4 (PDF/A-1). Ginebra: ISO 
ISO. 2006. ISO 23081-1:2006. Information and documentation. Records management processes. Metadata for records. Part 1: Principles. Ginebra: ISO.

ISO. 2008a. ISO 32000-1. Document management-Portable document format-Part 1: PDF 1.7. Ginebra. ISO

ISO. 2008b. ISO 26122. Information and documentation- Work process analysis for records. Ginebra. ISO.

ISO. 2009a. ISO 23081-2:2009. Information and documentation. Records management processes. Metadata for records. Part 2: Conceptual and implementation issues. Ginebra: ISO.

ISO. 2009b. ISO/TR 15801:2009 (2a rev.). Document management-Information stored electronically-Recommendations for trustworthiness and reliability. Ginebra: ISO.

ISO. 2009c. ISO 31000:2009. Risk. Management-Principles and guidelines. Ginebra. ISO.

ISO. 2010a. ISO 16175-1:2010. Information and documentation. Principles and functional requirements for records in electronic office environments. Part 1: Overview and statement of principles. Ginebra: ISO.

ISO. 2010b. ISO 16175-3:2010. Information and documentation. Principles and functional requirements for records in electronic office environments. Part 3: Guidelines and functional requirements for records in business systems. Ginebra: ISO.

ISO. 2010c. ISO/TR 13028:2010. Información y Documentación. Directrices para la implementación de la digitalización de documentos. Ginebra: ISO.

ISO. 2010d. ISO/TR 27003:2010 Information technology-Security techniques-Informatio security management systems-Guidance. Ginebra. ISO

ISO. 2011a. ISO 23081-3:2011. Information and documentation. Records management processes. Metadata for records. Part 3: Self-assessment method. Ginebra: ISO.

ISO. 2011b. ISO 16175-2:2011. Information and documentation. Principles and functional requirements for records in electronic office environments. Part 2: Guidelines and functional requirements for digital records management systems. Ginebra: ISO.

ISO. 2011c. ISO/30300:2011. Management system for records. Fundamentals and vocabulary. Ginebra: ISO.

ISO. 2011d. ISO/30301:2011. Management system for records. Requirements. Ginebra: ISO.

ISO. 2011e. ISO /IEC. 2011. Information technology -- Security techniques -- Information security risk management. Ginebra. ISO.

ISO. 2012a. ISO 19011:2012. Guidelines for quality and/or environmental management systems auditing. Ginebra: ISO

ISO. 2012b. ISO 13008:2012. Digital records conversion and migration process. Ginebra: ISO.

ISO. 2012c. ISO 14721:2012. Space data and information transfer systems - Open archival information system (OAIS). Reference model. Ginebra: ISO. (Norma UNE-ISO 14721:2015).

ISO. 2012d. ISO 16363:2012. Requirements for assessing the trustworthiness of digital repositories. Ginebra: ISO.

ISO. 2012e. ISO/TR 17068:2012. Information and documentation. Trusted third party repository for digital records. Ginebra: ISO.

ISO. 2012f. ISO 14641-1:2012. Electronic archiving- Part 1. Specifications concerning the design and the operation of an information system for electronic information preservation. Ginebra: ISO. (Norma UNE-ISO 14641-2015) 
ISO. 2012g. ISO 22301 Societat Security-Business Continuity Management-Systems/ Requirements. Ginebra. ISO.

ISO. 2013. ISO 27001:2013. Information technology. Security techniques. Information security management systems. requirements. Ginebra: ISO.

ISO. 2014a. ISO 27000:2014. Information technology-Security techniques-Information security management systems- Overwieu and vocabulary. Ginebra: ISO.

ISO. 2014b. ISO 16919:2014. Space data and information transfer systems. Requirements for bodies providing audit and certification of candidate trustworthy digital repositories. Ginebra: ISO.

ISO. 2014c. Borrador ISO/CDTR 17911:2014. Guidelines for long term preservation file format selection and conversion methodology. Ginebra: ISO.

ISO. 2014d. ISO/TR 18128: 2014. Information and Documentation. Risk assessment for records processes and systems. Ginebra. ISO.

ISO. 2015a. ISO 17021-1:2015. Conformity assessment. Requirements for bodies providing audit and certification of management systems. Ginebra: ISO.

ISO. 2015b. ISO 30302:2015. Information and documentation. Management systems for records. Guidelines for implementation. Ginebra: ISO.

ISO. 2015c. ISO/IEC/27006. 2015 Information technology -- Security techniques -- Requirements for bodies providing audit and certification of Information security management systems. Ginebra. ISO.

ISO. 2015d. ISO 9000:2015 Quality management systems -Fundamentals and vocabulary. Ginebra. ISO.

ISO. 2015e. ISO 9001:2015. Quality management systems-Requirements. Ginebra. ISO.

ISO. 2016a. ISO 15489-1:2016. Information and documentation -- Records management -- Part 1: Concepts and principles. Ginebra: ISO.

ISO. 2016b. ISO /IEC/ 27004. 2016 Information technology -- Security techniques -- Information security management -- Monitoring, measurement, analysis and evaluation. Ginebra. ISO.

Llanes-Padrón, D. y Moro-Cabero, M. 2015. La Archivística del canon. Presencia y presciencia en la formación universitaria de Brasil y España. Actas del IV Seminario Hispano-Brasileiro de Pesquisa em Informação, Documentação e Sociedade. (Brasil). Universidad de Brasilia, 113-136.

Lemieux, V. L. 2004. Managing risks for records and information. Lenexa: Arma.

Library of Congress. S.f. METS. Metadata encoding \& Transmission Standard. Fecha de consulta: 8 de febrero de 2018. http://www.loc.gov/standards/mets/

Marín Agudelo, S. 2012. "Estado de la Archivística en América Latina 200-2009. Perspectiva teórica y aproximación conceptual”. Investigación Bibliotecológica 26 (57): 77-101.

Moreiro-González, J. A. 2015. "Al hilo de las noticias sobre planes de estudio y mercado de trabajo”. Iwetel (THINKEPI), 7 de febrero de 2015. Fecha de consulta: 27 de junio de 2016. https//listserv.rediris.es/cgi-bin/wa?a2=ind1502A\&L=IWETEL \&P=IWETEL $\& 9=\mathrm{A} \& \mathrm{I}=3 \& \mathrm{~J}=$ on $\& \mathrm{~d}=$ Ninguna + coincidencia $\% 3 \mathrm{BCoinci}-$ dir\%3BCoincidencias $\& z=4$

National Council on Archives. 1997. Rules for the Construction of Personal, Place and Corporate Names. UK: National Council on Archives. Fecha de consulta: 30 de junio de 2015. http://archiveshub.ac.uk/ncarules/ 
NODAC: Norma de Descripción Archivística de Cataluña. 2007. Barcelona: Ministerio de Comunicación, Departamento de Cultura.

NOGADA: Norma Gallega de Descripción Archivística. 2008. Galicia: Junta de Galicia, Subdirección General del Patrimonio Cultural.

Norma Española. 2005. UNE-EN-ISO 19005-1: 2005. Gestión de documentos. Formato de fichero de documentos electrónicos para conservación a largo plazo. Parte 1. Uso de PDF.1.4. Madrid: Aenor.

Norma Española. 2008. UNE-ISO 9001:2015. Sistemas de gestión para la calidad.

Requisitos. Madrid: Aenor.

Norma Española. 2009 UNE-ISO 9004:2009. Gestión para el éxito sostenido de una organización. Enfoque de gestión de la calidad. Madrid: Aenor.

Norma Española. 2010. UNE-ISO-IN 73:2010. Gestión del riesgo. Vocabulario. Madrid: Aenor.

Norma Española. 2012. UNE-ISO 19011: 2012. Directrices para la auditoría de los sistemas de gestión de la calidad y/o ambiental. Madrid: Aenor.

Norma Española. 2013a. UNE-ISO 31010: 2013. Gestión del riesgo. Técnicas de apreciación del riesgo. Madrid: Aenor.

Norma Española. 2013b. UNE-ISO/IEC 27002:2013. Tecnología de la información-Técnicas de seguridad-Código de prácticas para los controles de seguridad de la información. Madrid. Aenor.

Norma Española. 2015a. UNE-EN-ISO 22301. Protección y seguridad de los ciudadanos. Sistema de gestión de la continuidad del negocio: especificaciones. Madrid: Aenor.

Norma Española. 2015b. UNE-EN-ISO 22302. Protección y seguridad de los ciudadanos.

Sistema de gestión de la continuidad de negocio: directrices. Madrid: Aenor.

Norma Española. 2015c. UNE-ISO 14721:2015. Sistemas de transferencia de datos e información espaciales. Sistema abierto de archivo de información (OAIS). Modelo de referencia. Madrid. Aenor.

Norma Española. 2017b. UNE-EN-ISO 27002:2017. Tecnologías de la Información-Técnicas de Seguridad. Código de prácticas para el control de la seguridad de la información. Madrid: Aenor.

NUDA: Norma Uruguaya de Descripción Archivística. 2014. Montevideo: Archivo General de la Nación.

Ortiz-Repiso, V. 2008. "Repensar els estudis de Biblioteconomia i Documentació a Espanya: traspassant els limites". Bid. Textos universitaris de Biblioteconia i Documentació 35. Fecha de consulta: 27 de junio de 2016. http://bid.ub.edu/es/35/ortiz.htm

PREMIS. 2008 . Data dictionary for preservation metadata. Fecha de consulta: 8 de febrero de 2018. https://www.loc.gov/standards/premis/

Rodríguez García, A. 2012. "Claves para la implementación de los lineamientos recursos, descripción y acceso”. Investigación Bibliotecológica 26 (56): 159-179.

Santamaría Gallo, A. 2006. La Norma Española de Descripción Archivística (NEDA): Análisis y propuesta de desarrollo. Madrid: Subdirección General de los Archivos Estatales.

Smit, F. 2013. "The metamorphosis of the records manager". Comma 2: 59-72.

Society of American Archivists. 2013. Describing Archives: A Content Standard, 2a. ed. Chicago: Society of American Archivists. Fecha de consulta: 30 de junio de 2015. http://files.archivists.org/pubs/DACS2E-2013.pdf 
Trust Worthy. 2007. "Repositories Audit \& Certification”. Criteria and Cheklist, 1. Fecha de consulta: 28 de abril de 2015. http://www.crl.edu/archiving-preservation/digital-archives/certification-and-assessment-digital-repositories

Unesco/UBC (The University of British Columbia). 2012. Vancouver Declaration. The memory of the world in the digital age: digitization and preservation. $5 \mathrm{p}$. Fecha de consulta: 8 de febrero de 2018. http://www.unesco.org/fileadmin/MULTIMEDIA/HQ/CI/CI/pdf/mow/unesco_ubc_vancouver_declaration_en.pdf

Para citar este texto:

Moro Cabero, Manuela y Dunia Llanes Padrón. 2018. "La importancia de la normalización para el ejercicio profesional del archivista”. Investigación Bibliotecológica: archivonomía, bibliotecología e información 32 (74): 193-223.

http://dx.doi.org/10.22 201/iibi.24488321xe.2018.74.57919 